\title{
Elliptic flow fluctuations in heavy ion collisions and the perfect fluid hypothesis
}

\author{
Sascha Vogel, Giorgio Torrieri, Marcus Bleicher \\ Institut für Theoretische Physik, J.W. Goethe Universität, Max von Laue-Straße 1, \\ 60438 Frankfurt am Main, Germany torrieri@fias.uni-frankfurt.de
}

(Dated: March 12, 2007)

\begin{abstract}
We analyze the recently measured $v_{2}$ fluctuation in the context of establishing the degree of fluidity of the matter produced in heavy ion collisions. We argue that flow observables within systems with a non-negligible mean free path should acquire a "dynamical" fluctuation, due to the random nature of each collision between the system's degrees of freedom. Because of this, $v_{2}$ fluctuations can be used to estimate the Knudsen number of the system produced at the relativistic heavy ion collider (RHIC). To illustrate this quantitatively, we apply the UrQMD model, with scaled cross sections, to show that collisions at RHIC have a Knudsen number at least one order of magnitude below the expected value for an interacting hadron gas. Furthermore, we argue that the Knudsen number is also bound from below by the $v_{2}$ fluctuation data, because too small a Knudsen number would break the observed scaling of $v_{2}$ fluctuations due to the onset of turbulent flow. We propose, therefore that $v_{2}$ fluctuation measurements, together with an understanding of the turbulent regime for relativistic hydrodynamics, will provide an upper as well as a lower limit for the Knudsen number. We also argue that an energy scan of $v_{2}$ fluctuations could shed light on the onset of the fluid regime.

PACS numbers: 25.75.-q,25.75.Dw,25.75.Nq
\end{abstract}

One of the most widely cited news (both in the academic and popular press) coming out of the heavy ion community concerns the discovery of a "perfect fluid" in collisions of heavy ions at RHIC [1-5]. The evidence for this claim comes from the successful modeling of the anisotropic expansion of the matter in the early stage of the reaction by means of ideal hydrodynamics $[\underline{6}-\underline{8}]$. This argument is compounded by the sensitivity of anisotropic expansion to shear viscosity [9, 10]. The presence of a non-negligible shear viscosity, therefore, can be detected by a careful analysis of anisotropic expansion data.

However, apart from this evidence for a small viscosity many fundamental properties of the fluid are unknown. E.g. it is currently discussed whether the observed fluid is a strongly interaction Quark-Gluon Plasma (sQGP) [11], a bound state Quark-Gluon Plasma (bsQGP) 12] or a (turbulent) Glasma [13] with instabilities [14, 15].

The connection between theory and experiment rests mainly on a single observable for the anisotropic expansion, namely the elliptic flow coefficient $v_{2}$. The parameter $v_{2}$ is the second Fourier component of the azimuthal anisotropy of the particle momenta given by [16, 17]

$$
v_{2} \equiv\left\langle\cos \left[2\left(\phi-\Phi_{\mathrm{RP}}\right)\right]\right\rangle \quad,
$$

where $\phi$ denotes the azimuthal angle of one outgoing particles and $\Phi_{R P}$ is the azimuthal angle of the reaction plane. The angular brackets denote an average over all considered particles from all events.

It should be stressed that (differential) studies of the collective flow are among the earliest predicted observable to probe heated and compressed nuclear matter [18]. As the transverse flow is intimately connected to the pressure gradients in the early stage of the reaction, it provides information on the equation of state (EoS) and might therefore be used to search for abnormal matter states and phase transitions [19 21].
The elliptic flow is of special importance, because it is "self-quenching" [16, 17, 22]: The angular pressure gradients creating the anisotropy extinguish themselves shortly after the start of the hydrodynamic evolution. Thus, the final $v_{2}$ is insensitive to later stages of the evolution, providing a key hole to the hottest, best thermalized, and possibly deconfined phase of the reaction.

In this paper we use the recently measured event-byevent fluctuations of $v_{2}[23,24$ to further investigate the properties of the fluid created at RHIC energies. The experimental data suggests that the $v_{2}$ fluctuations follow the fluctuations in initial eccentricity $\epsilon$

$$
\omega_{v_{2}}=\sqrt{\frac{\left\langle\left(\delta v_{2}\right)^{2}\right\rangle}{\left\langle v_{2}\right\rangle^{2}}}=\sqrt{\frac{\left\langle(\delta \epsilon)^{2}\right\rangle}{\langle\epsilon\rangle^{2}}} .
$$

This relation follows in a straight-forward fashion from the proportionality between $v_{2}$ and the eccentricity inferred from ideal [6 6 8] and viscous [9] boost-invariant hydrodynamics

$$
v_{2}=\beta \epsilon
$$

where $\beta$ is approximately constant (Indeed, since $\epsilon$ is a small dimensionless parameter driving anisotropy, this relation can be understood simply in terms of Taylor expansion).

As long as we are far away from the turbulent regime (on which we comment later), the deterministic nature of hydrodynamics,its applicability event by event and Eq. 3 constrain [25] the effect hydrodynamic propagation has on initial state fluctuations to the form

$$
\left.\delta v_{2}\right|_{\text {initial }} \sim \beta \delta \epsilon
$$

Explicit calculations have confirmed that this is the case for ideal hydrodynamics [26]. A non-zero viscosity should 
not alter the proportionality, but just lower the value of $\beta[25]$.

It is, however, surprising that initial conditions be the only source of fluctuations. If the system is treated as a collection of interacting particles, the random nature of each interaction should add a dynamical component to the fluctuation of any flow variable, which depends not on the initial conditions (with which it is not correlated) but on the random nature of each microscopic collision

$$
\left\langle\left(\delta v_{2}\right)^{2}\right\rangle \simeq \beta^{2}\left\langle(\delta \epsilon)^{2}\right\rangle+\Delta_{d y n}^{2}
$$

One can quantify the degree of perfection of the fluid by the Knudsen number 25] defined as:

$$
K n=\frac{\lambda}{L} \simeq \frac{N_{\text {particles }}}{N_{\text {collisions }}}
$$

with $\lambda$ the mean free path of the particles, $L$ the typical length scale of the system $N_{\text {particles }}$ the total number of particles and $N_{\text {collisions }}$ denoting the total number of interactions (soft and hard).

If the Knudsen number is zero, the system becomes a perfect fluid. In this case, flow observables are fully deterministic. Hence, the probability of a $v_{2}$ at a certain time assuming a given eccentricity is a $\delta$ - function.

$$
P\left(v_{2} \mid \epsilon\right) \sim \delta\left(v_{2}-\beta \epsilon\right)
$$

and hence $\Delta_{d y n}$ is zero.

In the limit of large numbers of collisions, correlations between collisions become weak, so the probability distribution in Eq. 7 becomes

$$
P\left(v_{2} \mid \epsilon, K n\right) \sim \frac{1}{2 \pi \sigma} e^{-\left(v_{2}-\beta \epsilon\right)^{2} / 2 \sigma^{2}}
$$

where $\sigma(K n)$ goes to 0 as the Knudsen number goes to zero. Thus, it is sensible to Taylor expand around $K n$, so

$$
\Delta_{d y n} \sim \alpha \sqrt{K n}+\mathcal{O}\left(K n^{2}\right)
$$

Note that the only "small parameter" here is the Knudsen number. All dependence on the nature of degrees of freedom and their interactions (in particular, whether the particles interacting are hadrons or partons, what is the equation of state etc.) is encoded within the parameter $\alpha$, which by naturalness is of order unity. In the case of a vanishing Knudsen number (the ideal hydrodynamic limit) $\Delta_{d y n}$ should vanish.

Such a scaling, apparent in Kinetic theory, can be also derived within hydrodynamics [27]: Fluctuations in fluids include a thermal fluctuation term (irrelevant here since $v_{2}$ is defined in a way that makes it independent of random multiplicity fluctuations) and a dynamical autocorrelation of the energy-momentum tensor. This autocorrelation scales linearly with the shear and bulk viscosity, which in turn depend linearly on the mean free path 27] and the inverse of the typical number of collisions per particle. Subsequent developments [28, 29] have not altered these basic conclusions, which have also been compared to Boltzmann equation simulations [30]. The latter comparison makes us confident of the "universality" of our scaling, since hydrodynamic fluctuations treated in [27, 29, 30] concern systems where the Boltzmann equation fails (e.g. water).

This is important, since the definition in Eq. 6 looks more natural within a Boltzmann equation formulation, which is in turn based on the scattering approximation between interactions. It is not clear whether this is a good approximation to use within RHIC. While some groups have managed to bring models based on these assumptions in agreement with RHIC data 31], the appearance of fields and off-shell effects in the strongly coupled limit is not unreasonable 32. This matter is complicated by the fact that everyday fluid 1 (where compressibility is typically large and correlated with viscosity due to Pauli blocking effects) are fundamentally different from ultrarelativistic ones (where the Pauli principle is not thought to be relevant, compressibility is bound by causality and typically unrelated to viscosity). In the latter, the everyday definition of fluid vs gas (based on compressibility) becomes inapplicable, and a small viscosity alone is no guarantee that the Boltzmann equation approximation is not a good one.

Our definition of Knudsen number, however, is general enough to be independent of these considerations: The Knudsen number is simply the ratio of a microscopic scale (where quantum randomness is important) to the macroscopic scale, in this case the total size of the system. It is easy to see that, to leading order, the ratio of the two quantities must generally be $\sim \eta /(s T L) \sim K n$ [33]. Such a definition of the Knudsen number allows us to recover the scaling found in [27 29] (derived with systems where the Boltzmann equation is not a good approximation, such as water, in mind).

It is therefore apparent that $\omega_{v_{2}}$ is a test for the hypothesis that the system at RHIC is a "perfect fluid", i.e. a locally thermalized system, where "many" particles undergo "many" collisions over a "small" fraction of the system's evolution. Potentially, this test is considerably more model-independent than a hydrodynamic analysis of $\left\langle v_{2}\right\rangle$, since $\Delta_{d y n}$ scales directly with the Knudsen number, and all other factors are of order unity.

Deviations from this limit, including plasma instabilities, or clustering, should therefore contribute fluctuations to $v_{2}$ [34] that can be probed by comparison to the newly available experimental data. Interestingly, the "opposite" limit to hydrodynamics, a classical nonAbelian field such as the "color glass condensate" (CGC) [35], is also fully deterministic, and hence would exhibit $\Delta_{d y n}=0$. Just like ideal hydrodynamics, however, the

\footnotetext{
1 The usual linguistic usage is "liquid" for the definition based on compressibility, and "fluid" for the definition based on viscosity. These two are often used as synonym in everyday English.
} 
absence of dynamical fluctuations in the CGC is an artifact of it being an effective description with a zero "small parameter". Here, the "small parameter" giving rise to fluctuations would be the inverse of the occupancy number of each quantum state. As the initial occupancy number at RHIC is 3-4 [35], diminishing to $\leq 1$ as the CGC melts, we expect large dynamical fluctuations also in a CGC prethermal stage, through calculating them is best left to a future work.

In this work, we quantitatively assess the sensitivity of $\omega_{v_{2}}$ on $K n$ by a string/hadron transport approach, the UrQMD v2.3 model [36, 37]. To explore the different regimes, we rescale the total interaction cross sections by factors of $1 / 2,1$ and 3 to vary the strength of the interaction.

Note that we are not using this rescaled UrQMD as a realistic model of the system, but rather as a "toy model" to study the scaling of $v_{2}$ fluctuations of the Knudsen number. We believe this is an appropriate approach for the present study because the results can, in a straightforward fashion, be converted into an estimate for the Knudsen number in heavy ion collision at RHIC. We expect that an analysis with partonic degrees of freedom will yield the same scaling with the Knudsen number, and a quantitative result within the same order of magnitude, the differences being encoded in the constant $\alpha$ of $\mathrm{Eq} 9$,

As UrQMD is a quantum molecular dynamics simulation, the Knudsen number can be effectively "measured" by keeping track of the collisions during the system's lifetime, with $K n \sim\left\langle N_{\text {particles }} \mid N_{\text {collisions }}\right\rangle \sim 0.6-1.5$. This analysis parametrically agrees with an estimate following [25], from the ratio of the calculated elliptic flow to the hydrodynamic expectation.

UrQMD also accounts for the expected non-flow effects, as well as the fluctuations in the initial condition, that also contribute to $\omega_{v_{2}}$ [38]. For a general discussion of the $v_{2}$ analysis within this approach the reader is referred to 39, 40].

The results of the present calculations are shown in Fig. 1. As can be seen, $\omega_{v_{2}}$ and $K n$ have the expected qualitative dependence on the over-all scaling parameter: As the factor used to rescale interaction cross sections increases, $\left\langle v_{2}\right\rangle$ increases [38] and $\omega_{v_{2}}$ decreases. However, both $\left\langle v_{2}\right\rangle[38]$ and $\omega_{v_{2}}$ are well away from the data-points even if the cross section is increased by a factor of three. Beyond the given increase, we run into technical difficulties and grossly over-estimate the total multiplicity of the system. Hence, we are not able to explore the scaling of the cross-section further than three times the physical one within the present approach.

Fig. 2 shows the scaling of $\omega_{v_{2}}$ w.r.t. the Knudsen number. The full line shows a fit of the calculations assuming a the additional fluctuations can be modeled by Eqs. 5] and 9, with $\alpha$ and $\beta$ are extracted from the fit. While $\alpha$ varies with the fundamental properties of the system even in the Poissonian limit, the fitted value is sufficient for an order of magnitude estimate of $\mathrm{Kn}^{-1}$.
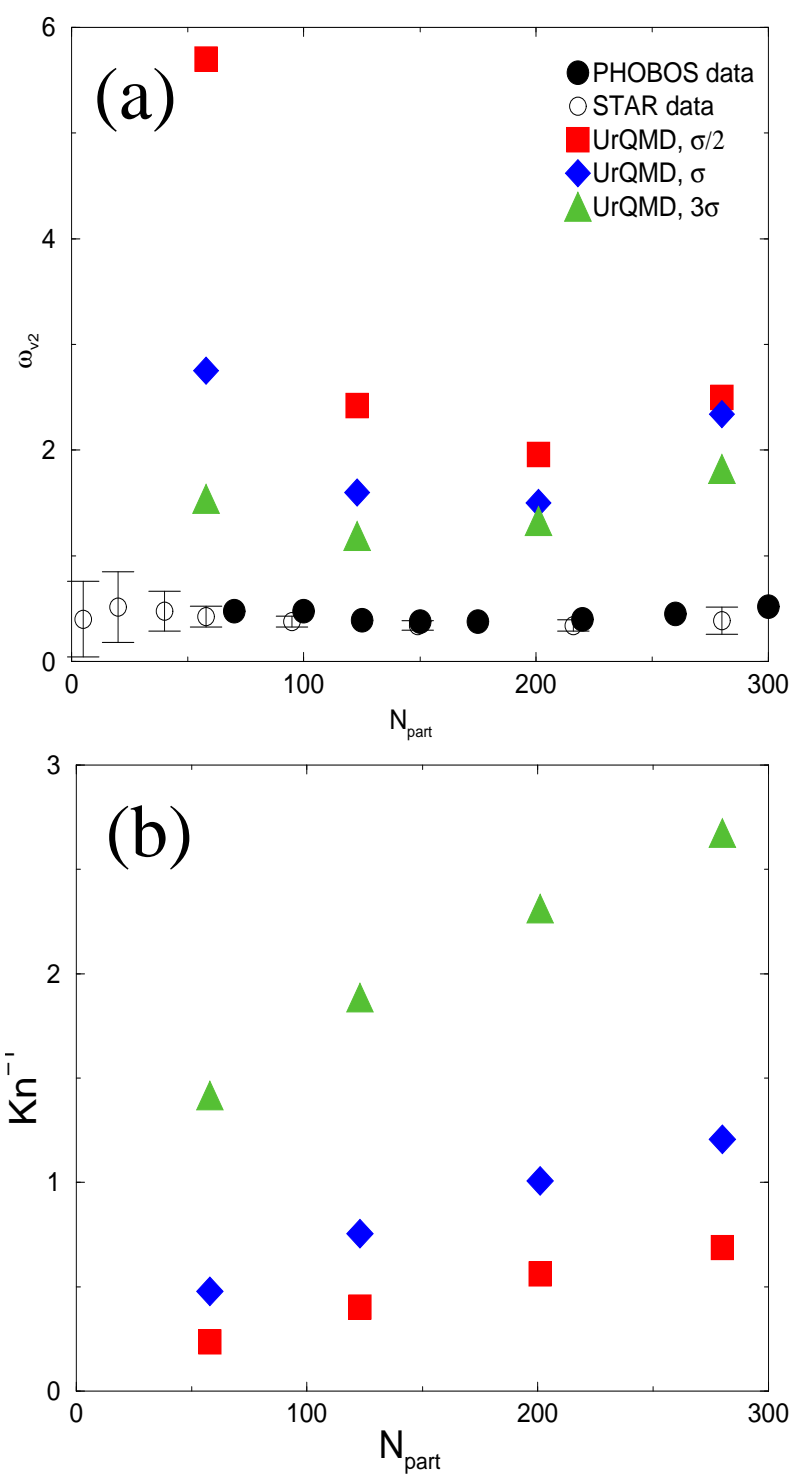

FIG. 1. (color online) UrQMD result for $\omega_{v_{2}}$ (panel (a)) and the Knudsen number (panel (b)) as a function of the number of participants for $\mathrm{Au}+\mathrm{Au}$ reaction at $\sqrt{s}_{N N}=200 \mathrm{GeV}$. The data are taken from [23, 24].

The scaling in Fig. 2 can be used to extrapolate to the inverse Knudsen number needed to describe the elliptic flow fluctuations observed at RHIC. Extrapolating the dependence of Eq. 9 to the upper experimental error bar for $\omega_{v 2}$ yields a lower bound on the inverse Knudsen number on the order of a hundred. This estimate for the Knudsen number (or the potential scaling factor for the cross section, as the Knudsen number is inversely proportional to the cross section) qualitatively agrees, with the opacity estimate derived from $\left\langle v_{2}\right\rangle$ using $\mathrm{pQCD}$ transport calculations [41, 42], as well as with the estimate obtained through transverse momentum fluctuations [43].

With the present calculation, we have established an upper limit to the Knudsen number. It is natural to ask 
what happens to $\omega_{v_{2}}$ as the Knudsen number goes to zero, and the system is more closely approximated by ideal hydrodynamics.

The Knudsen number is related to another well known number in hydrodynamics, namely Reynold's number. Reynold's number is defined as

$$
R e=\frac{H L\langle v\rangle}{\eta}=\frac{s T L\langle v\rangle}{\eta} \sim 3 \frac{\langle v\rangle}{K n},
$$

with $H$ being the enthalpy, $\eta$ being the viscosity, $s$ being the entropy density, $T$ denoting the temperature and $\langle v\rangle$ the typical flow velocity. Thus, a small Knudsen number goes hand in hand with an increase of Reynold's number.

However, too high Reynolds numbers inevitably lead to instabilities of the hydrodynamic flow (the turbulent regime) and will add an additional source of fluctuations to $\omega_{v_{2}}$, due to instabilities in the flow formation. Estimating the Reynold's number for the present transport simulations leads to $R e \sim 1$. However using the presently advocated ADS/CFT bound $\eta / s=1 / 4 \pi$ 44] leads to Reynolds numbers well into the $\sim 10^{2}$ in the initial stages of the hydrodynamic evolution (Fig. 3). for $T=200 \mathrm{MeV}, L=10 \mathrm{fm}$ and $\langle v\rangle \sim 1 / \sqrt{3}$ (the speed of sound for a relativistic ideal gas), we have $R e \sim 100$.

Following [27 hydrodynamic instabilities will be present starting from $R e=10-100$. If $R e>100-1000$ the flow will generally become turbulent 27], although the onset of turbulence will also depend on the boundary conditions: the larger the bluffness of a layer of fluid (defined by $\left\langle\left|u^{i} \times \overrightarrow{d A}\right|\right\rangle$, where $u^{i}$ is the flow vector and $\overrightarrow{d A}$ the layer surface element), the less Reynolds number is required for the onset of turbulence. For a compressible fluid expanding from an "almond-like" shock, the last condition is likely to be satisfied close to the "edges" of the almond, provided that compressibility does not quench the onset of turbulence (the last question is not conclusively settled, through recent evidence [45, 46] suggests that adding compressibility does not significantly change the critical Reynolds number for the onset of turbulence).

Thus, we are led to conclude that, below a certain critical Knudsen number, the scaling in Eq. [5 should break down and $\omega_{v_{2}}$ should increase significantly above the "ideal" Eq. 2 value: While $\Delta_{d y n}$ would continue to decrease with decreasing Knudsen number, $\omega_{v_{2}}$ would not anymore scale with $\left\langle(\delta \epsilon)^{2}\right\rangle /\langle\epsilon\rangle$ but the initial fluctuation would be amplified by the turbulent evolution. If $\tau$ is the lifetime of the system and $\tau_{0}$ the timescale of the evolution of turbulence, $\omega_{v_{2}}$ in a turbulent fluid would scale as an exponent of an approximately power-law function of the initial volume (i.e. the number of participants)

$$
\left.\omega_{v_{2}} \sim \frac{\left\langle\left(\delta v_{2}\right)^{2}\right\rangle}{\left\langle v_{2}\right\rangle}\right|_{\tau \ll \tau_{0}} e^{\tau / \tau_{0}} \sim \frac{\left\langle(\delta \epsilon)^{2}\right\rangle}{\langle\epsilon\rangle} \exp \left[N_{\text {part }}^{\kappa}\right]
$$

From causality (the time it takes for rarefaction waves to travel across the system, $\sim$ size $/ c_{s} \sim N_{\text {part }}^{1 / 3} / c_{s}$, where $c_{s}$ is the speed of sound), it can be deduced that $\kappa \simeq 1 / 3$.

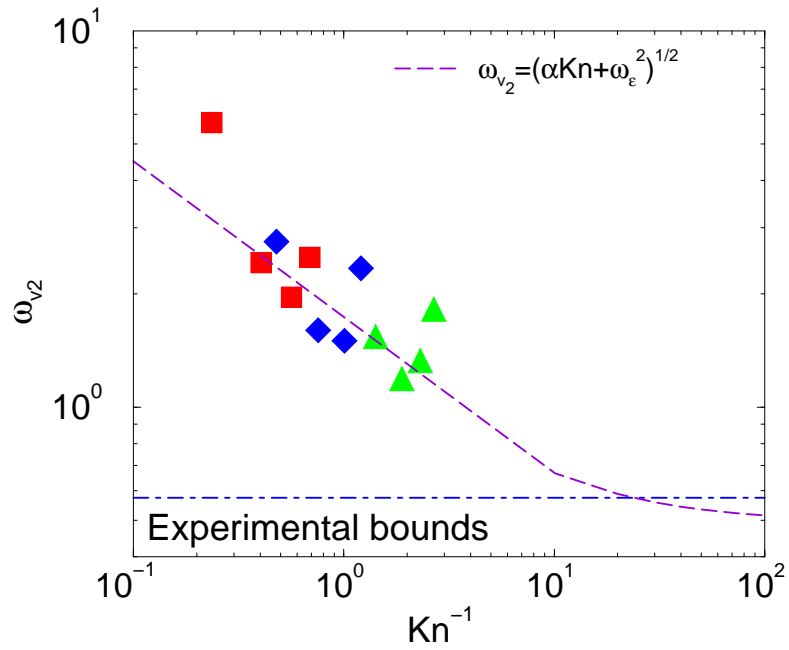

FIG. 2. (color online) Relationship between $\omega_{v_{2}}$ and the Knudsen number, plotted together with the Poissonian expectation. The parameter $\alpha$ was fitted from the data. See Fig. 1 for the legend

It is apparent from Fig. 1 that such a scaling is not observed in the experimental data 23, 24], so the viscosity of the system created at RHIC is high enough to place it out of the turbulent regime. This sustains the argument that the mere observation of a well-defined $\left\langle v_{2}\right\rangle$ places a lower constraint on viscosity because it signals that the system is not in a sufficiently turbulent regime.

The implications of this statement on the closeness of the fluid created at RHIC to the ADS/CFT viscosity bound are still not clear. It is difficult to make a more precise estimate since the turbulence in the system produced in heavy ion collisions has not as yet been studied (for first attempts with QCD transport approaches, the reader is referred to [14, 15, 47, 48]).

In one dimension, the stability of boost-invariant dynamics (the boundary condition used,either exactly or in approximate form for simulations at RHIC energy) has been thoroughly studied. Boost-invariant evolution was found to be generally stable at the early stages, where $v_{2}$ forms [49, 50] (through instabilities could play a big role during freeze-out [51, 52]). This leads us to think that if the system does have an early turbulent stage, $\tau_{0}$ remains long compared to its duration. This conclusion is however bound to change within full 3D hydrodynamics, especially if the system is not to a good approximation boost invariant, as recent initial state calculations suggest 47, 48.

Thus, before a quantitative answer to these questions can be given, a transport or hydrodynamic model capable of modeling turbulence at the scale of heavy ion collisions, and hence of inferring a quantitative value of $\tau_{0}$ in Eq. 11, is necessary. Up to now, the only known calculation of the Reynolds number and the onset of turbulent flow in heavy ion collision has been done in Ref. [53].

The onset of turbulence could be signaled experimen- 


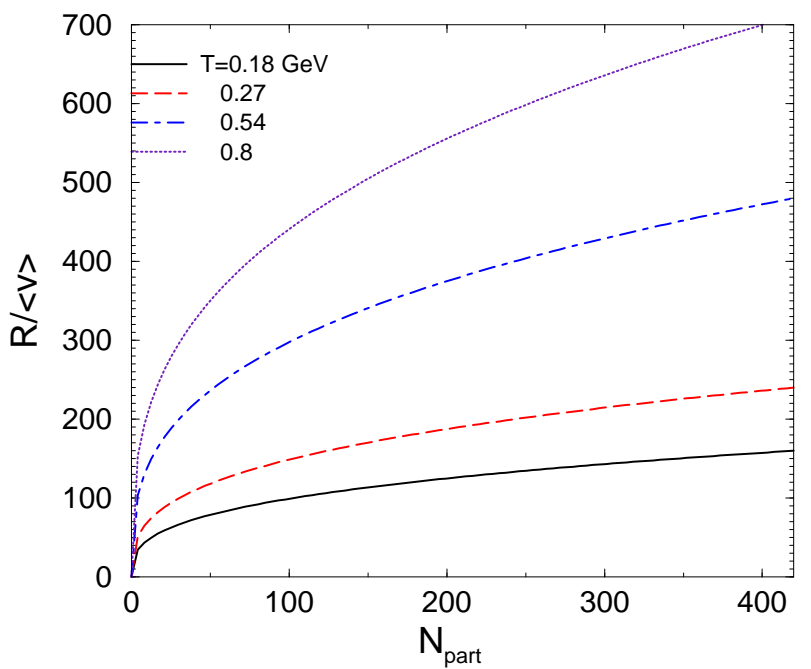

FIG. 3. (color online) The Reynolds number of a system the size of a collision between nuclei $A$, with the viscosity given by the conjectured ADS/CFT universal bound.

tally by a widening of $\omega_{v 2}$ and a change of its dependence on $N_{\text {part }}$ from constant to exponential scaling as per Eq. 11. Thus, combined with the data in the non-turbulent regime, analyzed using the ansatz of Eq. [5] the experimental measurement of $\omega_{v_{2}}$ in a wide range of energies and system sizes can yield a lower as well as an upper limit of $K n$.

Moreover, the energy dependence of $\omega_{v_{2}}$ could acquire a crucial phenomenological role in the light of the universal scaling seen in $v_{2} / \epsilon$ 54 56] (panel (a) in Fig. 4). The scaling variable is the multiplicity rapidity density normalized by the initial overlap surface $d N /(S d y)$, chosen because it corresponds, in the boost-invariant picture [58], to the entropy density divided by thermalization time. One could interpret this scaling as the approach to the ideal hydrodynamics limit as the initial density become large. If this interpretation is correct, the Knudsen number smoothly decreases as an inverse power of $d N /(S d y)$, but has little sensitivity to the change in degrees of freedom at the phase transition [57]. Alternatively, it could be that the large flow observed at RHIC is indicative of a downward "jump" in the Knudsen number when the critical initial density needed to free partonic degrees of freedom is achieved.

The observation of $\omega_{v_{2}}$, and the excitation function of $\omega_{v_{2}} / \omega_{\epsilon}$ could differentiate between these scenarios (Fig. 4 panel (b)). If the system smoothly becomes more fluid at greater density, $\omega_{v_{2}} / \omega_{\epsilon}$ can be expected to decrease inversely with $d N /(S d y)$ (smoothly if this is a continuous approach to hydrodynamics or abruptly if the a transition to a more fluid regime is linked to a phase transition). If fluidity is present in all systems to the same amount, $\omega_{v_{2}} / \omega_{\epsilon}$ will stay constant across energies and system sizes.

In conclusion, we have argued that the experimental

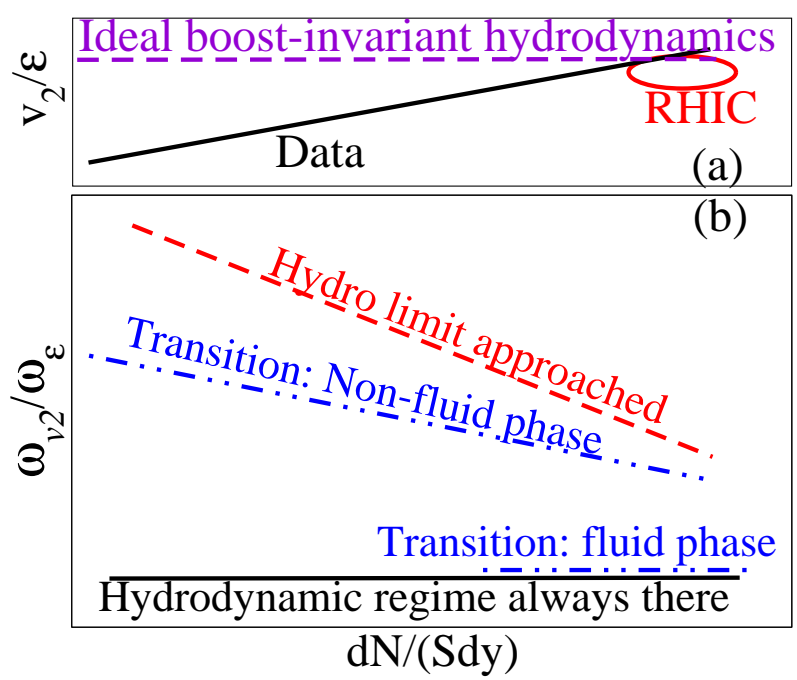

FIG. 4. (color online) A qualitative plot (panel (b)) showing the scaling of the $v_{2}$ fluctuations in the three scenarios suggested by the scaling of $v_{2}$ across energy and system size 54 57, shown in panel (a). The $x$-axis, the rapidity density normalized by the overlap area, corresponds to the entropy density in the Bjorken hydrodynamic scenario. If the hydrodynamic limit is smoothly approached with increasing system volume/lifetime the difference between the observed $v_{2}$ fluctuation and the initial estimate should go as the red dashed line. If the hydrodynamic limit indicates a transition between a viscous hadronic gas and the sQGP, the scaling with $1 / S d N / d y$ should be broken, with higher energy ("sQGP regime") lower centrality events having a lower $v_{2}$ fluctuation than equivalent more central ("hadronic regime") events. This is indicated in the plot by the blue dot-dashed line. Finally, a constancy of $\omega_{v 2} / \omega_{\epsilon}$ might indicate that the "hydro regime" was actually with us all along, and only the initial conditions are responsible for the apparent rise in $v_{2}$.

observation of $\omega_{v_{2}}$ can provide unique information to estimate the Knudsen number $K n$, and hence to to pin down the perfection of the fluid created in heavy ion collisions quantitatively. We have used a transport model to estimate a lower limit of $\mathrm{Kn}^{-1}$, and found that it is nearly two orders of magnitude below the value needed to describe the $v_{2}$ fluctuations at RHIC. We have also argued that the currently observed scaling of $\omega_{v_{2}}$ should break in the turbulent regime, and hence the measurement of $\omega_{v_{2}}$ potentially places an upper as well as a lower limit on $K n^{-1}$. We have furthermore suggested that, in the light of these considerations, an energy and system size scan of $v_{2}$ fluctuations can shed light on the approach to the hydrodynamic regime. However, before these limits can be quantitatively ascertained, much more theoretical modeling and experimental investigation is required.

The computational resources have been provided by the Center for Scientific Computing, CSC at Frankfurt University. G.T. was (financially) supported by the Helmholtz International Center for FAIR within the framework of the LOEWE program (Landesoffensive 
zur Entwicklung Wissenschaftlich-konomischer Exzellenz) launched by the State of Hesse. We wish to thank
H. Stoecker, Art Poskanzer, C. Greiner, M. Gyulassy, J. Rafelski,R. Venugopalan and S. Jeon for fruitful discussions.

[1] Scientific American, May 2006, [28] L.P.Csernai, Zs.Lazar,I.A.Lazar,D.Molnar, http://www.sciam.com/article.cfm?chanID=sa006\&articleID=00019Ripilek,D.D.Strottman, Phys. Rev. E 61237 (2000) 037F-1448-837F83414B7F014D\&pageNumber=1\&catID=2 [29] D.N.Zubarev， V.G.Morozov， Physica 120A 411-467 New York Times, http://www.nytimes.com/2005/04/19/science/1918p.3.html BNL Press release, http://www.bnl.gov/bnlweb/pubaf/pr/PR3djspldy_arpciarMDAM0H438 sour, G.C.Lie,M.Mareschal,E.Clementi,

[2] I. Arsene et al. [BRAHMS Collaboration], Nucl. Phys. A 757, 1 (2005) arXiv:nucl-ex/0410020.

[3] B. B. Back et al., Nucl. Phys. A 757, 28 (2005) arXiv:nucl-ex/0410022.

[4] J. Adams et al. [STAR Collaboration], Nucl. Phys. A 757, 102 (2005) arXiv:nucl-ex/0501009.

[5] K. Adcox et al. [PHENIX Collaboration], collaboration," Nucl. Phys. A 757, 184 (2005) arXiv:nucl-ex/0410003.

[6] P. F. Kolb and U. W. Heinz, arXiv:nucl-th/0305084.

[7] D. Teaney, J. Lauret and E. V. Shuryak, Phys. Rev. Lett. 86, 4783 (2001) arXiv:nucl-th/0011058.

[8] P. F. Kolb, U. W. Heinz, P. Huovinen, K. J. Eskola and K. Tuominen, Nucl. Phys. A 696, 197 (2001) arXiv:hep-ph/0103234.

[9] P. Romatschke and U. Romatschke, Phys. Rev. Lett. 99, 172301 (2007) arXiv:0706.1522 [nucl-th]].

[10] D. Teaney, Phys. Rev. C 68, 034913 (2003) arXiv:nucl-th/0301099.

[11] M. Gyulassy, arXiv:nucl-th/0403032

[12] E. Shuryak, Prog. Part. Nucl. Phys. 53, 273 (2004) arXiv:hep-ph/0312227.

[13] T. Lappi and L. McLerran, Nucl. Phys. A 772, 200 (2006) arXiv:hep-ph/0602189.

[14] S. Mrowczynski, Phys. Lett. B 314 (1993) 118.

[15] P. B. Arnold and G. D. Moore, Phys. Rev. D 73, 025006 (2006)

[16] J. Y. Ollitrault, Phys. Rev. D 46, 229 (1992).

[17] A. M. Poskanzer and S. A. Voloshin, Phys. Rev. C 58, 1671 (1998) arXiv:nucl-ex/9805001.

[18] W. Scheid, J. Hofmann and W. Greiner, In *Berkeley 1974, Proceedings, Lawrence Berkeley Lab Lbl-3675*, Berkeley 1974, 1-50.

[19] H. Stöcker, J. A. Maruhn and W. Greiner, Z. Phys. A 290 (1979) 297.

[20] J. Hofmann, H. Stöcker, U. W. Heinz, W. Scheid and W. Greiner, Phys. Rev. Lett. 36 (1976) 88.

[21] H. Stöcker and W. Greiner, Phys. Rept. 137 (1986) 277.

[22] H. Sorge, Phys. Rev. Lett. 78, 2309 (1997) arXiv:nucl-th/9610026.

[23] B. Alver et al. [PHOBOS Collaboration], Phys. Rev. Lett. 104, 142301 (2010) arXiv:nucl-ex/0702036.

[24] P. Sorensen [STAR Collaboration], J. Phys. G 35, 104102 (2008) [arXiv:0808.0356 [nucl-ex]].

[25] R. S. Bhalerao, J. P. Blaizot, N. Borghini and J. Y. Ollitrault, Phys. Lett. B 627, 49 (2005) arXiv:nucl-th/0508009.

[26] R. Andrade, F. Grassi, Y. Hama, T. Kodama and O. . J. Socolowski, Phys. Rev. Lett. 97, 202302 (2006) arXiv:nucl-th/0608067.

[27] Landau, L.D. and Lifshitz, E.M. (1987) "Fluid Mechanics", Pergamon Press (1987)
[31] Z. Xu, C. Greiner and H. Stocker, Phys. Rev. Lett. 101, 082302 (2008) arXiv:0711.0961 [nucl-th]].

[32] W. Cassing and E. L. Bratkovskaya, Phys. Rev. C 78, 034919 (2008) arXiv:0808.0022 [hep-ph]].

[33] B. Betz, D. Henkel and D. H. Rischke, J. Phys. G 36 (2009) 064029.

[34] S. Mrowczynski and E. V. Shuryak, Acta Phys. Polon. B 34, 4241 (2003) arXiv:nucl-th/0208052.

[35] F. Gelis, E. Iancu, J. Jalilian-Marian and R. Venugopalan, arXiv:1002.0333 [hep-ph].

[36] M. Bleicher et al., J. Phys. G 25, 1859 (1999) arXiv:hep-ph/9909407.

[37] S. A. Bass et al., Prog. Part. Nucl. Phys. 41, 225 (1998) arXiv:nucl-th/9803035.

[38] X. l. Zhu, M. Bleicher and H. Stoecker, Phys. Rev. C 72, 064911 (2005) arXiv:nucl-th/0509081.

[39] M. Bleicher and H. Stoecker, Phys. Lett. B 526, 309 (2002) arXiv:hep-ph/0006147.

[40] H. Petersen, Q. Li, X. Zhu and M. Bleicher, Phys. Rev. C 74, 064908 (2006) arXiv:hep-ph/0608189.

[41] D. Molnar and M. Gyulassy, Nucl. Phys. A 697, 495 (2002) [Erratum-ibid. A $\mathbf{7 0 3 ,} 893$ (2002)] arXiv:nucl-th/0104073.

[42] D. Molnar and P. Huovinen, Phys. Rev. Lett. 94, 012302 (2005) arXiv:nucl-th/0404065.

[43] S. Gavin and M. Abdel-Aziz, Phys. Rev. Lett. 97, 162302 (2006) arXiv:nucl-th/0606061.

[44] P. Kovtun, D. T. Son and A. O. Starinets, Phys. Rev. Lett. 94, 111601 (2005) arXiv:hep-th/0405231.

[45] Lechner R., Sesterhenn, J., Friedrich, R. , J. Turbulence 2:1-25, (2001).

[46] Huang P., Coleman G., Bradshaw P. J. Fluid Mech 305:185-218, (1995).

[47] A. Dumitru and Y. Nara, Phys. Lett. B 621, 89 (2005) arXiv:hep-ph/0503121.

[48] M. Strickland, arXiv:hep-ph/0701238

[49] H. Kouno, M. Maruyama, F. Takagi and K. Saito, Phys. Rev. D 41, 2903 (1990).

[50] W. Florkowski, B. L. Friman, G. Baym and P. V. Ruuskanen, Nucl. Phys. A 540, 659 (1992).

[51] G. Torrieri, B. Tomasik and I. Mishustin, Phys. Rev. C 77, 034903 (2008) arXiv:0707.4405 [nucl-th].

[52] G. Torrieri and I. Mishustin, Phys. Rev. C 78, 021901 (2008) arXiv:0805.0442 [hep-ph].

[53] G. Baym, Nucl. Phys. A 418 (1984) 525C.

[54] D. J. Hofman [the PHOBOS Collaboration], arXiv:nucl-ex/0701051

[55] S. A. Voloshin, arXiv:nucl-ex/0701038

[56] G. Torrieri, Phys. Rev. C $\mathbf{7 6}, 024903$ (2007) arXiv:nucl-th/0702013. 
[57] G. Torrieri, arXiv:0911.4775 [nucl-th].

[58] J. D. Bjorken, Phys. Rev. D 27, 140 (1983). 